\title{
Evaluación de una marca desde la perspectiva del consumidor y su relación con la perdurabilidad empresarial ${ }^{1}$
}

\author{
Evaluation of a brand from the consumer's perspective \\ and its relationship with business sustainability
}

Como citar este artículo / To reference this articcle:

Forero-Casas Laura A.' Otero-Gómez María C. \& Giraldo-Pérez Wilson (2016). Evaluación de una marca desde la perspectiva del consumidor y su relación con la perdurabilidad empresarial EL CONUCO: (investigación, economía y sociedad), 3(1), pp. 1-8.

DOI: https://doi.org/10.22579/2619614X.529

Forero-Casas Laura A. *

Otero-Gómez María C. **

Giraldo-Pérez Wilson ***

\begin{abstract}
Artículo de investigación
Recepción: 8/23/2019

Aceptación: 12/20/2019

El Conuco es una revista de acceso abierto revisada por pares. (C) $2018 \mathrm{El}$ autor (es). Este es un artículo de acceso abierto distribuido bajo los términos de la Licencia Internacional Creative Commons Attribution 4.0 (CC-BY 4.0), que permite el uso, distribución y reproducción sin restricciones en cualquier medio, siempre que se acredite el autor y la fuente originales.

Consulte http://creativecommons.org/licenses/by/4.0/.
\end{abstract} ๑OPEN ACCESS

(c) $($ i) $\Theta(?$

(c) ${ }_{\mathrm{BY}} \mathrm{NC}_{\mathrm{ND}}$

* Estudiante de Administración de Empresas, Universidad de los Llanos. Email: laura.forero.casas@unillanos.edu.co Código ORCID: https://orcid.org/0000-0003-3310-8424

** Magíster en Mercadeo, profesora asociada Facultad de Ciencias Económicas, Universidad de los Llanos, líder grupo de investigación Dinámicas de Consumo. Email: motero@ unillanos.edu.co, Código ORCID: https://orcid.org/00000002-3241-7877

*** Magíster en Mercadeo, profesor asistente, Facultad de Ciencias Económicas, Universidad de los Llanos, integrante del grupo de investigación Dinámicas de Consumo. Email: wgiraldo@unillanos.edu.co, Código ORCID: https://orcid. org/0000-0001-8514-9805

\section{Resumen}

Este manuscrito tiene como objetivo determinar la influencia del valor de marca en las intenciones de recompra de un producto de tal forma que contribuya en la perdurabilidad empresarial. El estudio se apoya en los conceptos generales del valor de marca con sus dimensiones: lealtad, notoriedad, calidad percibida, asociaciones y comportamiento del mercado. El estudio empírico utiliza un enfoque cuantitativo en el que se procesan los datos procedentes de 321 encuestas. Se analizan los resultados de las correlaciones entre las dimensiones del valor de marca con la variable dependiente intención de recompra. Los resultados sugieren que existe una relación positiva, pero con un valor estadístico medio, lo que indica que estas dos variables están significativamente relacionadas. Los datos revelan que la dimensión que presenta una relación mas alta es la correspondiente a las asociaciones, por el contrario, la variable con menor relación es la lealtad. Se concluye que el conocimiento de las percepciones de los consumidores sobre las marcas, permite la adopción de medidas conducentes a la perdurabilidad empresarial en el largo plazo.

Palabras Clave. Consumidor, perdurabilidad empresarial, marca.

1 Este artículo se deriva de la opción de grado titulada "El aporte del valor de marca a la perdurabilidad empresarial a través de la intención de recompra", se enmarca en la clasificación de pasantía en proyectos de investigación y se vincula al proyecto titulado "Antecedentes y consecuencias del valor de marca. Un estudio centrado en los consumidores jóvenes colombianos", que cuenta con el aval de la Dirección General de Investigaciones (DGI) de la Universidad de los Llanos y se circunscribe al grupo de investigación Dinámicas de Consumo de la Escuela de Administración y Negocios de la Facultad de Ciencias Económicas. 


\section{Abstract}

This manuscript aims to determine the influence of brand equity on the repurchase intentions of a product in such a way that it contributes to business sustainability. The study is based on the general concepts of brand equity with its dimensions: loyalty, notoriety, perceived quality, associations and market behaviour. The empirical study uses a quantitative approach in which data from 321 surveys are processed. The results of the correlations between the dimensions of brand value with the dependent variable repurchase intention are analyzed. The results of the study suggest a positive relationship, but with a statistical mean value, indicating that these two variables are significantly related. The data reveal that the dimension with the highest relationship is the one corresponding to the associations, while the variable with the lowest relationship is loyalty. It is concluded that the knowledge of the consumers' perceptions about brands, allows the adoption of measures leading to business sustainability in the long term.

Keywords. Consumer, business sustainability, brand.

JEL: M10, M29, M30

\section{Introducción}

La literatura científica indica que las marcas se han convertido en activos fundamentales para las empresas, de allí el interés por medir y gestionar su desempeño. Para los gerentes, comprender la forma como los consumidores valoran sus marcas, es un insumo invaluable porque aporta al desarrollo de estrategias de marca exitosas (Baalbaki \& Guzmán, 2016). Es decir, una marca puede servir como un mecanismo alternativo a través del cual aumenta o disminuye el rendimiento de la empresa. Si bien, las marcas usualmente se relacionan con las interpretaciones que hace el consumidor en torno al simbolismo que representa, dado el nivel de relevancia y diferenciación que trae implícito su uso, también está el análisis desde el punto de vista empresarial. Por ejemplo, Morgan (2012) indica que las marcas pueden ser valiosos recursos intangibles que permiten a las empresas construir y proteger su participación en el mercado, siempre y cuando el valor de marca sea fuerte y positivo.

Asimismo, una de las expectativas que se generan cuando se crea una empresa, es su perdurabilidad en el tiempo, que se relaciona con la capacidad de las organizaciones para alcanzar y superar los objetivos financieros establecidos. Por tanto, es necesario adaptarse a las características de los diferentes agentes económicos y a las exigencias de las fuerzas del mercado. Para adaptarse a estas fuerzas y tener resultados financieros superiores, las empresas cuentan con productos identificados con sus marcas, cuya finalidad se centra en la diferenciación en el mercado. Esta diferenciación se refleja en el incremento de las preferencias de los consumidores, dicho de otro modo, la marca contribuye al aumento del volumen de las ventas, y en consecuencia en el fortalecimiento del flujo de caja a largo plazo, además de la obtención de un mejor apalancamiento comercial (Chiu, Huang, Weng \& Chen, 2017).

A pesar de la existencia de trabajos empíricos que analizan el valor de marca y su relación con las intenciones de recompra (Reddy \& Kavitha, 2020), la investigación sobre el impacto de estas variables en la perdurabilidad empresarial es prácticamente nula. Por tanto, este manuscrito, proporcionan una iluminación modesta a la literatura de gestión de marketing, particularmente en el contexto de las empresas dedicadas al diseño y la manufactura de morrales, ropa y accesorios en Colombia. El objetivo de este estudio consiste en 
determinar la influencia del valor de marca en las intenciones de recompra de un producto de tal forma que contribuya en la perdurabilidad empresarial Siendo así, surgen los siguientes interrogantes ¿De qué manera influye el valor de marca en las intenciones de recompra de una marca? ¿Qué estrategias se deben implementar para que las intenciones de recompra contribuyan en la perdurabilidad empresarial? Para dar respuesta a estas cuestiones se tomará el aporte teórico de Aaker (1996), debido a que este autor expone el concepto del valor de marca desde la perspectiva del consumidor y desde la organización.

\section{Revisión de literatura}

Kotler \& Armstrong (2013) definen el marketing como "un proceso social y directivo mediante el que los individuos y las organizaciones obtienen lo que necesitan y desean a través de la creación y el intercambio de valor con los demás” (p.5), este planteamiento sugiere que a nivel general, las estrategias empresariales se deben encaminar hacia la creación de valor tanto para la oferta como como para la demanda. Actualmente, estas estrategias empresariales han evolucionado al punto que han impactado significativamente en el consumidor, cuyos resultados se convierten en experiencias de vida para ellos, siendo este el valor añadido en la forma como las empresas venden sus productos. Al respecto, Aaker (1996) hace un llamado a que los resultados de las estrategias deben "reflejar el valor de los activos de la marca y centrarse en una ventaja sostenible que los competidores no puedan duplicar fácilmente" (p. 3).

Así que, el valor que tiene una marca frente al mercado es de vital importancia, ya que de esta depende su perdurabilidad en el tiempo; en consecuencia, todos los procesos organizacionales tanto internos como externos deben encaminarse al alcance de este objetivo. De esta manera,
Rivera \& Cardona (2012) destacan que para que una empresa sea perdurable debe, entre otros aspectos: ser rentable a través del tiempo; adaptarse a las condiciones cambiantes del medio; y enfrentarse a la competencia optimizando la cadena de valor. En este sentido, los autores también indican que la perdurabilidad se relaciona con la capacitación permanente y la innovación, con el fin de que la organización sea altamente competitiva y pueda generar ventajas frente al mercado. Estas ventajas pueden asociarse con la rentabilidad de las ventas, siendo así un elemento fundamental para la supervivencia y el crecimiento de las organizaciones comerciales.

Sin embargo, es necesario precisar que para identificar o medir el grado de rentabilidad de las ventas, lo primero que se debe hacer es evaluar las características de la estrategia comercial implementada. En este caso, un aspecto que ha tomado fuerza en la literatura del marketing es el análisis de la marca desde la perspectiva del consumidor. Se ha demostrado que las marcas altamente valoradas por los clientes generan múltiples beneficios para la empresa, esto se debe a que "las ventas se correlacionan directamente con el valor de marca” (Ortegón, 2013, p.20).

De este modo, el valor de marca se convierte en una variable importante dentro de la estrategia comercial, debido a que una empresa difícilmente puede perdurar en el mercado si sus marcas carecen de reconocimiento. Sumado a esto, la competencia se convierte en una fuerte amenaza, principalmente si cuenta con recursos que administrados adecuadamente pueden conllevar a opacar o liquidar tanto a las marcas como a las empresas rivales. Aunque existen diversos autores que mencionan varios elementos que conformar el valor de marca, para efectos de esta investigación se tendrán en cuenta los postulados de Aaker (1996) debido a que este autor vincula 
el valor de marca desde la perspectiva del consumidor y desde la organización. Así, desde el enfoque del consumidor se analizan varias dimensiones como la lealtad, la notoriedad, la calidad percibida y las asociaciones; y desde el punto de vista de la organización están las medidas de comportamiento del mercado (Aaker, 1996). A continuación, se presentan los conceptos de Aaker (1996) para describir cada una de las dimensiones que conforman el valor de marca:

- La lealtad está relacionada con el precio premium y la satisfacción. El precio premium es la cantidad de dinero que está dispuesto a pagar una persona por un producto/servicio; $y$, la satisfacción es la experiencia que tienen los consumidores con productos y servicios.

- La notoriedad es la rápida identificación de los productos por parte de los consumidores al estar en un mercado, de esto dependen sus características, funciones y principalmente la forma como se vende el producto.

- La calidad percibida se relaciona con la experiencia obtenida por el consumidor con un producto/servicio y su posterior intención de repetir la compra, de esto depende que la marca tenga aceptación y crecimiento.

- Las asociaciones las componen el valor percibido, la personalidad de marca y la marca como organización.

- El comportamiento de mercado está definido por los resultados y datos que arrojan los estudios de mercado, que luego sirven para realizar estadísticas y definir las variables de un producto a partir del análisis de los precios, la competencia, la distribución, la aceptación de un producto, entre otras.

Otro concepto que se aborda en esta investigación es la intención de recompra, puesto que el interés del consumidor por volver a adquirir pro- ductos de la misma marca permite a las empresas hacer una aproximación a la proyección en sus ventas. En tal sentido, se presenta el concepto que tiene como finalidad la retención de los clientes, así:

- Intención de recompra: Es la voluntad de un consumidor de volver a comprar a la misma empresa, el mismo o diferente producto (Yang, Huang, Liang \& Huang, 2017).

\section{Metodología}

En los siguientes párrafos se detallan los procedimientos y las técnicas utilizadas durante la recogida, el procesamiento y el análisis de la información.

\section{Contexto de estudio}

Los datos objeto de análisis se recogieron mediante la aplicación de una encuesta que se distribuyó tanto de forma física como a través de los entornos online. La única característica que debían cumplir los encuestados es que sean compradores, usuarios o influenciadores de la decisión de compra de la marca objeto de análisis. Las personas que respondieron el formulario residen en diferentes ciudades de Colombia.

\section{Muestra}

Para el desarrollo de esta investigación cuantitativa se consideró como nicho objetivo a estudiantes universitarios con edades entre los $16 \mathrm{y}$ 24 años. Se entrevistaron a 321 jóvenes, entre las características sociodemográficas se destaca que el 46\% pertenece al segmento masculino. La distribución por grupo etario es la siguiente: de 18 a 22 años (75\%), entre 16 y $17(14 \%)$ y mayores de $22(11 \%)$. En una etapa previa a la recolección de la información se explicó a los participantes que se trata de una encuesta completamente anónima, por lo que no se recoge ninguna información 
que pueda identificarlos individualmente. En todos los casos se atendieron los protocolos establecidos por el Comité de Bioética de la Universidad de los Llanos, tanto en los jóvenes adultos como en los menores de edad.

\section{Diseño de la investigación}

Para el análisis de la demanda se diseñó un cuestionario estructurado con el fin de reunir la información necesaria para alcanzar los objetivos del estudio. Se optó por la implementación de un muestreo por conveniencia. Las variables analizadas se midieron usando escalas adaptadas de la literatura científica (Lassar, Mittal \& Sharma, 2005; Yoo, Donthu \& Lee, 2000; Netemeyer, et al, 2004; Pappu, Quester y Cooksey, 2005). En todos los ítems los participantes informaron su nivel de identificación para cada declaración en una escala Likert de 5 puntos $(1=$ Totalmente en desacuerdo y $5=$ Totalmente de acuerdo). Para el procesamiento y análisis de los datos se utilizó el software libre JASP versión 0.9.2.0

\section{Análisis de resultados}

Teniendo en cuenta que el interés de este estudio se enfocó en identificar cuál es el aporte del valor de marca a la perdurabilidad empresarial a través de la intención de recompra, en la tabla 1 se presenta la relación entre estas variables. Los datos revelan que existe una relación positiva, pero con un valor estadístico medio, lo que indica que estas dos variables están significativamente relacionadas. Dando como resultado que las dimensiones de valor de marca sí influyen en la intención de recompra.

Tabla 1. Correlación entre variables valor de marca e intención de recompra

\begin{tabular}{lccc}
\hline & & $\begin{array}{c}\text { Intención de } \\
\text { recompra }\end{array}$ & p-value \\
\hline Valor de marca & Spearman's rho & 0,636 & $<0,001$ \\
\hline
\end{tabular}

Fuente: Elaboración propia, 2020.
La tabla 2 demuestra que las dimensiones notoriedad, calidad percibida, asociaciones organizacionales y lealtad tienen una relación positiva y significativamente estadística con el valor de marca; la relación más alta es con la dimensión asociaciones que alcanza un 0,902 en la cual se encuentran variables estudiadas como el valor percibido, la personalidad de la marca y la credibilidad de la organización. Por su parte, la dimensión lealtad presenta un 0,632 siendo el valor más bajo respecto a las demás dimensiones, lo que indica que la empresa debe realizar mayores esfuerzos en su gestión de marca ante los consumidores, debido a que es más conveniente retener a sus clientes actuales, en lugar de buscar nuevos compradores.

Tabla 2. Correlaciones entre las dimensiones de la variable valor de marca

\begin{tabular}{|c|c|c|c|}
\hline & & $\begin{array}{c}\text { Valor de } \\
\text { marca }\end{array}$ & p-value \\
\hline Notoriedad & \multirow{4}{*}{ Spearman's rho } & 0,687 & $<0,001$ \\
\hline Calidad percibida & & 0,793 & $<0,001$ \\
\hline $\begin{array}{c}\text { Asociaciones } \\
\text { organizacionales }\end{array}$ & & 0,902 & $<0,001$ \\
\hline Lealtad & & 0,632 & $<0,001$ \\
\hline
\end{tabular}

Fuente: Elaboración propia, 2020.

La tabla 3 muestra la relación entre cada una de las dimensiones del valor de marca y la intención de recompra, las cuales presentan resultados significativos y de estos la lealtad es el factor con la fuerza de asociación más alta siendo 0,599.

Tabla 3. Correlaciones entre las dimensiones y la variable intención de recompra

\begin{tabular}{|c|c|c|c|}
\hline & & $\begin{array}{c}\text { Intención de } \\
\text { recompra }\end{array}$ & p-value \\
\hline Notoriedad & \multirow{4}{*}{ Spearman's rho } & 0,360 & $<0,001$ \\
\hline Calidad percibida & & 0,523 & $<0,001$ \\
\hline $\begin{array}{c}\text { Asociaciones } \\
\text { organizacionales }\end{array}$ & & 0,504 & $<0,001$ \\
\hline Lealtad & & 0,599 & $<0,001$ \\
\hline
\end{tabular}

Fuente: Elaboración propia, 2020. 
De los datos anteriores, es de resaltar los buenos niveles de asociaciones que los consumidores tienen con la empresa dueña de la marca analizada. Lo que sugiere en términos de valor de marca, que la compañía brinda confianza, credibilidad y es admirada por los compradores de morrales, ropa y accesorios, quienes en el momento de realizar sus compras y recompras, son leales a la marca; en consecuencia, estos dos elementos se convierten en una ventaja competitiva en el mercado nacional.

En la tabla 4, las pregunta 1 y 2 se relacionan con la perdurabilidad empresarial, dado que en ellas se indaga a los entrevistados si seguirían comprando la marca en el futuro. Las respuestas arrojan resultados estadísticamente significativos, con dirección positiva y altamente correlacionados.

Tabla 4 Correlaciones entre las preguntas y la dimensión intención de recompra

\begin{tabular}{|c|c|c|c|}
\hline & & $\begin{array}{l}\text { Intención de } \\
\text { recompra }\end{array}$ & $\mathrm{p}$-value \\
\hline $\begin{array}{l}\text { Seguiré pagando por } \\
\text { productos de esta marca }\end{array}$ & & 0,911 & $<0,001$ \\
\hline $\begin{array}{l}\text { Tengo la intención de } \\
\text { seguir comprando esta } \\
\text { marca en el futuro }\end{array}$ & Spearman's rho & 0,914 & $<0,001$ \\
\hline $\begin{array}{c}\text { Seguiría comprando } \\
\text { productos de esta marca } \\
\text { en lugar de cualquier otra }\end{array}$ & & 0,906 & $<0,001$ \\
\hline
\end{tabular}

Fuente: Elaboración propia, 2020.

Según la conceptualización de Aaker (1996) y basados en los datos anteriores, se puede inferir que el factor de la gestión de marca con mayor incidencia en la perdurabilidad empresarial corresponde a las asociaciones organizacionales. Se trata de un resultado valioso para la compañía, puesto que los consumidores perciben a su marca como "una marca fuerte", por la que expresan lealtad y una propensión a repetir las compras en el futuro; esto permite a la compañía realizar su proyección en ventas lo que indica un camino sólido hacia la perdurabilidad empresarial.

Continuando con este razonamiento, a continuación se da respuesta al interrogante ¿De qué manera influye el valor de marca en las intenciones de recompra de una marca? De los resultados se deduce que debido a que la lealtad es una variable clave en la retención de clientes, se deben aumentar y reforzar las estrategias de fidelización otorgando beneficios, esto en atención a que son determinantes en la intención de recompra por parte de los consumidores a lo largo de todos los meses del año.

Estos hallazgos conllevan al desarrollo de propuestas que contribuyan en la perdurabilidad empresarial, al respecto se infiere que las estrategias que más se ajustan son las asociadas a la innovación. Según Collins y Porras (Como se citó en Ollé, Planellas, Torres, Urriolagoitia \& Sorribas, 2009) "las compañías que gozan de un éxito continuado poseen valores y un propósito centrales que permanecen invariables en el tiempo mientras que sus estrategias de negocio van adaptándose a un mundo cambiante" (p.14); estas estrategias son las indicadas porque al ser innovadores estarán abiertos al cambio y de esta forma podrán desarrollar una visión que sea adecuada para alcanzar su perdurabilidad.

Finalmente, para responder el objetivo general que busca determinar la influencia del valor de marca en las intenciones de recompra de un producto de tal forma que contribuya en la perdurabilidad empresarial, se establece que el valor de marca es una variable clave y esta correlacionada directamente con las ventas (Ortegón, 2013). Entonces, para que las empresas puedan lograr resultados financieros superiores, deben focalizar sus estrategias organizacionales con miras a que su valor de marca sea fuerte y de esta manera 
puedan perdurar en el mercado tal como lo indican Rivera \& Cardona (2012).

\section{Conclusiones}

De acuerdo con los resultados, se puede concluir que el valor de marca es un factor importante y tiene una relación positiva con la intención de recompra, es decir que, respecto al valor de marca, una empresa que añade estrategias organizacionales tanto interna (perspectiva organización) como externamente (perspectiva del consumidor), tiene mayores probabilidades de perdurar en el mercado (Aaker, 1996). Asimismo, la experiencia del consumidor con la marca es elemento que aumenta o disminuye la probabilidad de la intención de recompra.

Con lo anterior, se demuestra que la perdurabilidad empresarial está sujeta a la intención de recompra, debido a que las dimensiones contenidas en el valor de marca fueron medidas de acuerdo con la experiencia como cliente o conocedor de la marca, en su mayoría dieron resultados significativamente altos, siendo esto un factor positivo en la intención de recompra. De igual manera, la perdurabilidad empresarial se da solo si la organización añade estrategias que permitan un estado de constante innovación y eficiencia, como son: adecuaciones en tiendas físicas y virtuales, evaluaciones de la experiencia post venta, innovación y mejoras en los productos, evaluaciones respecto a la atención al cliente en tiendas físicas y virtuales, desarrollo de nuevos mercados y cobertura en nichos desatendidos, entre otros.

Finalmente, la perdurabilidad empresarial puede verse influenciada por el valor de marca que tienen los consumidores desde sus perspectivas; situación bastante favorable y en armonía con lo expresado por Ortegón (2013), en el sentido de que las ventas guardan una correlaciona directa con el valor de marca. Es decir que, si el valor de marca es alto, las ventas tienden a incrementarse, desencadenando en la rentabilidad, lo que conlleva a la perdurabilidad empresarial. Así pues, el valor de marca es un determinante que debe ser objeto de estudio en las organizaciones para lograr su perdurabilidad y lograr su sostenimiento en un mercado volátil y cambiante como el actual. 


\section{Referencias}

Aaker, D. (1996). Measuring brand equity across products and markets. California Management Review, 38 (3), 102-120.

Baalbaki, S. \& Guzmán, F. (2016). A consumer-perceived consumer-based brand equity scale. Journal of Brand Management, 23(3), 229-251.

Chiu, M., Huang, H., Weng, Y. \& Chen, Ch. (2017). The roles of customer-brand relationships and brand equity in brand extension acceptance. Journal of Electronic Commerce Research, 18(2), 155-176.

Lassar, W., Mittal, B. \& Sharma, A. (1995). Measuring customer- based brand equity. Journal of Consumer Marketing, 12 (4), 11-19.

Morgan, N.A. (2012). Marketing and business performance. Journal of the Academy of Marketing Science, 40, 102-119.

Netemeyer, R. G., Krishnan, B., Pullig, C., Wang, G., Yagci, M., Dean, D., Ricks, J. \& Wirth, F. (2004). Developing and validating measures of facets of customer-based brand equity. Journal of Business Research, 57(2), 209-224.

Ollé, M., Planellas, M., Torres, D., Urriolagoitia, L. \& Sorribas, M. (2009). Investigaciones previas. En D. Torres. (Ed.), Estudio sobre los factores de éxito de las grandes empresas de servicios en España (pp.11-21).
Ortegón, L. (2013). Relación entre valor de marca y las ventas. Un estudio aplicado en compañías agroquímicas. Revista Ciencias Estratégicas 21(29), 105-124.

Pappu, R., Quester, P. G. \& Cooksey, R. W. (2005). Comsumer - based brand equity: Improving the measurement - empirical evidence. Journal of Product and Brand Management, 14(3), 143-154.

Reddy, V. D. \& Kavitha, S. F. (2020). Impact of brand equity on patronage and repurchase intention of branded apparels in Chennai city. International Journal of Psychosocial Rebabilitation, 24 (1), 1611 1618.

Rivera, H. \& Cardona, D. (2012). Protocolo de investigación. Bogotá: Editorial Universidad del Rosario. Recuperado de https: / / repository.urosario.edu.co/flexpaper/ handle/10336/4179/Fasciculo145. pdf? ?equence $=1$ \&isAllowed $=\mathrm{y}$

Yang, F., Huang, M., Liang, C. \& Huang, C. (2017). A Study of the Relationships among Perceived Service Innovation, Flow Experience and Repurchase Intention. International Journal of Organizational Innovation, 10(2), 13-28.

Yoo, B., Donthu, N. \& Lee, S. (2000). An examination of selected marketing mix elements and brand equity. Journal of the Academy of Marketing Science, 28(2), 195-211 